Pacific Journal of Mathematic 


\title{
A CONDITIONAL ENTROPY FOR THE SPACE OF PSEUDO-MENGER MAPS
}

\author{
Alan SALESKI
}

\begin{abstract}
Let $X$ be a set and $T: X \rightarrow X$ be a bijection. Consider the space $\mathscr{H}$ of pseudo-Menger maps on $X$ which induce a compact topology on $X$ for which $T$ is a homeomorphism. The lattice properties of $\mathscr{C}$ are investigated and a bivariate nonnegative function of $\mathscr{H}$ is defined which possesses certain properties analogous to those of the usual conditional entropy function defined on the space of measurable partitions of a probability space.
\end{abstract}

1. Introduction. A pseudo-Menger map on a set $X$ is, roughly speaking, an assignment of a distribution function to every pair of points in $X$ in a manner consistent with the axioms of a pseudometric space. Each such map induces a topology on $X$ as defined by Schweizer and Sklar [9]. Let $T$ be a bijection of $X$ onto itself. Let $\mathscr{C}$ denote the space of all pseudo-Menger maps which induce a compact topology on $X$ for which $T$ is a homeomorphism. If $\theta \in \mathscr{M}$ let $h(T, \theta)$ denote the topological entropy of $T$ with respect to the topology on $X$ induced by $\theta$. In [7] it was shown that $h(T, \cdot)$ is leftcontinuous in the sense that if $\theta, \theta_{n} \in \mathscr{M}, \theta_{n} \geqq \theta$ and $\theta_{n}(p, q) \rightarrow \theta(p, q)$ in distribution for all $p, q \in X$ then $h\left(T, \theta_{n}\right) \rightarrow h(T, \theta)$. In an effort to extend this result one is led to ask the question whether $\mathscr{M}$ is closed under the operations of Max and Min and, if so, what can one say about the entropy of $T$ acting on the topology engendered by the maps obtained as a result of such operations. We now proceed to provide precise definitions and notation.

2. Preliminaries. Let $I$ denote the closed unit interval, $\boldsymbol{R}$ the real numbers and $\boldsymbol{Z}^{+}$the positive integers. Let $\mathscr{D}$ be the set of all left-continuous monotone increasing functions $F: R \rightarrow I$ satisfying $F(0)=0$ and $\sup _{x} F(x)=1$. Endowed with the Lévy metric $\mathscr{D}$ is a complete metric space. If $F_{n}, F \in \mathscr{D}$ then $F_{n} \stackrel{\mathscr{D}}{\rightarrow} F$ will denote convergence with respect to the Lévy topology. It is well-known that $F_{n} \stackrel{\mathscr{P}}{\rightarrow} F$ if and only if $F_{n}(x) \rightarrow F(x)$ for each $x \in R$ at which $F$ is continuous. If $F, G \in \mathscr{D}$ then $F \geqq G$ will mean $F(x) \geqq G(x)$ for all $x \in \boldsymbol{R}$. Let $H \in \mathscr{D}$ be the function defined by: $H(t)=0$ for $t \leqq 0$ and $H(t)=1$ for $t>0$.

Let $X$ be a fixed set. Let $\mathscr{F}(X)$ denote the collection of all functions $\theta: X \times X \rightarrow \mathscr{D}$. For convenience we shall often write $\theta_{p q}$ in place of $\theta(p, q)$. A statistical pseudo-metric space is an ordered pair $(X, \theta)$ where $\theta \in \mathscr{F}$ satisfies: 
(SM 1)

$$
\theta_{p q}=\theta_{q p} \quad \text { for all } p, q \in X
$$

(SM 2) $\theta_{p q}(a+b)=1$ whenever $\theta_{p r}(a)=\theta_{r q}(b)=1$ for some $r \in X$

$$
\theta_{p p}=H \text { for all } p \in X \text {. }
$$

If, in addition, $\theta$ satisfies:

$$
\theta_{p q}=H \quad \text { only if } \quad p=q
$$

then $(X, \theta)$ is a statistical metric space. Let $\mathscr{S}(X)$ denote the collection of all $\theta$ for which $(X, \theta)$ is a statistical pseudo-metric space.

A triangular norm is a function $\Delta: I \times I \rightarrow I$ which is associative, commutative, non-decreasing in each variable and satisfies $\Delta(y, 1)=y$ for each $y \in I$. A continuous Menger space [pseudo-Menger space] is a statistical metric space [statistical pseudo-metric space] $(X, \theta)$ for which there exists a continuous triangular norm $\Delta$ satisfying:

(SM 5) $\theta_{p r}(a+b) \geqq \Delta\left(\theta_{p q}(a), \theta_{q r}(b)\right)$ for all $p, q, r \in X$ and $a, b \in \boldsymbol{R}$.

Let $\mathscr{C}(X)$ denote the set of all $\theta$ for which $(X, \theta)$ is a continuous pseudo-Menger space. If $\theta_{n}, \theta \in \mathscr{M}(X)$ and $\theta_{n}(p, q) \stackrel{\mathscr{S}}{\rightarrow} \theta(p, q)$ for all $p, q \in X$ then we will write $\theta_{n} \stackrel{\mathscr{H}}{\rightarrow} \theta$. Similarly, if $\theta, \Gamma \in \mathscr{C}(X)$ and $\theta_{p q} \geqq \Gamma_{p q}$ for all $p, q \in X$ then we write $\theta \geqq \Gamma$. Let $\Xi \in \mathscr{C}(X)$ be defined by $\Xi_{p q}=H$ for all $p, q \in X$.

If $\theta \in \mathscr{F}(X)$ let $X$ be endowed with the topology, denoted $\tau(\theta)$, generated by all sets of the form $N(p, \varepsilon, \lambda, \theta)=\left\{q \in X: \theta_{p q}(\varepsilon)>1-\lambda\right\}$ where $p \in X, \varepsilon>0, \lambda>0$. Let $T: X \rightarrow X$ be a bijection. Let $\mathscr{L}(X, T)=\{\theta \in \mathscr{C}(X): T$ is a self-homeomorphism of $(X, \tau(\theta))$ and $\tau(\theta)$ is compact $\}$.

If $\theta \in \mathscr{L}(X, T)$ we will let $h(T, \theta)$ denote the topological entropy of $T$ with respect to the $\tau(\theta)$ topology. We will follow the notation and definitions of topological entropy developed in [1]. The only exception is the understanding that if $T$ is a self-homeomorphism of $(X, \tau)$ where $\tau$ is not a compact topology and $\mathscr{U} \subset \tau$ is a cover of $X$ which possesses a finite subcover then $h(T, \mathscr{C})=\lim _{k \rightarrow \infty}(1 / \mathrm{k}) H\left(\mathbf{V}_{j=0}^{k-1} T^{j} \mathscr{C}\right)$. We let $\mathscr{M}_{F}(X, T)=\{\theta \in \mathscr{M}(X, T): h(T, \theta)<\infty\}$.

Let $\rho$ be a pseudo-metric on a set $Y$ and let $\mathscr{C}$ be an open cover of $(Y, \rho)$. Then $\rho$-diam $\mathscr{W}$ will mean the $\sup \{\rho$-diam $A: A \in \mathscr{U}\}$. Let $\tau(\rho)$ denote the topology on $Y$ determined by $\rho$. In addition, if $\varepsilon>0$ and $a \in Y$ then let $B(Y, a, \rho, \varepsilon)=\{q \in Y: \rho(q, a)<\varepsilon\}$. If $D$ is another pseudo-metric on $Y$ then $\rho \geqq D$ will mean $\rho(a, b) \geqq D(a, b)$ for all $a, b \in Y$. Finally, if $X$ is a pseudo-metric space then $X^{*}$ will denote the (unique up to uniform isomorphism) completion for which $X^{*} \sim X$ is Hausdorff. Such an $X^{*}$ will be called the pseudo-metric space completion of $X$. 
3. Lattice operations. If $\theta, \Psi \in \mathscr{M}(X, T)$ define $\theta \vee \Psi=$ $\operatorname{Min}(\theta, \Psi)$ and $\theta \wedge \Psi=\operatorname{Max}(\theta, \Psi)$. It is easy to construct examples in which $\theta \wedge \Psi$ fails even to belong to $\mathscr{S}(X)$. However, we will show that $\theta \vee \Psi$ admits a canonical extension to a map belonging to $\mathscr{M}\left(X^{*}, T^{*}\right)$ where $X^{*}$ is the completion of $(X, \tau(\theta \vee \Psi))$ and $T^{*}$ is the extension of $T$ to $X^{*}$.

Proposition 1. If $\theta, \Psi \in \mathscr{M}(X)$ then $\theta \vee \Psi \in \mathscr{C}(X)$.

Proof. Let $\Delta_{1}$ and $\Delta_{2}$ be continuous triangular norms for $\theta$ and $\Psi$ respectively which satisfy:

$$
\theta_{p q}(a+b) \geqq \Delta_{1}\left(\theta_{p r}(a), \theta_{r q}(b)\right)
$$

and

$$
\Psi_{p q}(a+b) \geqq \Delta_{2}\left(\Psi_{p r}(a), \Psi_{r q}(b)\right) \text { for all } a, b \in \boldsymbol{R} \text { and all } p, q, r \in X \text {. }
$$

It is easy to check that $\Delta_{3}=\operatorname{Min}\left(\Delta_{1}, \Delta_{2}\right)$ is a continuous triangular norm. Using the monotonicity of $\Delta_{1}$ and $\Delta_{2}$ we verify the triangle inequality for $\theta \vee \Psi$ with respect to $\Delta_{3}$ :

$$
\begin{aligned}
(\theta \vee \Psi)_{p r}(a+b) & =\operatorname{Min}\left(\theta_{p r}(a+b), \Psi_{p r}(a+b)\right) \\
& \geqq \operatorname{Min}\left(\Delta_{1}\left(\theta_{p q}(a), \theta_{q r}(b)\right), \Delta_{2}\left(\Psi_{p q}(a), \Psi_{q r}(b)\right)\right) \\
& \geqq \operatorname{Min}\left(\Delta_{1}, \Delta_{2}\right)\left(\operatorname{Min}\left(\theta_{p q}(a), \Psi_{p q}(a)\right), \operatorname{Min}\left(\theta_{q r}(b), \Psi_{q r}(b)\right)\right) \\
& =\Delta_{3}\left((\theta \vee \Psi)_{p q}(a),(\theta \vee \Psi)_{q r}(b)\right) .
\end{aligned}
$$

Lemma 1. Assume $\theta, \Psi \in \mathscr{L}(X)$ determine compact topologies $\tau(\theta)$ and $\tau(\Psi)$ respectively. Let $d_{1}$ and $d_{2}$ be pseudo-metrics on $X$ which generate the topologies $\tau(\theta)$ and $\tau(\Psi)$ respectively. Then the pseudo-metric $D=d_{1}+d_{2}$ determines the topology $\tau(\theta \vee \Psi)$.

Proof. As a consequence of Lemma 4 of [7] and the above proposition we know that $\tau(\theta \vee \Psi) \supset \tau(\theta) \cup \tau(\Psi)$. Thus it suffices to show that $\tau(\theta \vee \Psi)$ is generated by $\{A \cap C: A \in \tau(\theta)$ and $C \in \tau(\Psi)\}$. Let $p \in X, \varepsilon>0$ and $\lambda>0$ be given. Let $q \in N(p, \varepsilon, \lambda, \theta \vee \Psi)$. For each $n \in Z^{+}$choose $A_{n}=N(q, 1 / n, 1 / n, \theta)$ and $C_{n}=N(q, 1 / n, 1 / n, \Psi)$. Suppose for each $n$ there exists $y_{n} \in A_{n} \cap C_{n}$ such that $y_{n} \notin N(p, \varepsilon, \lambda, \theta \vee \Psi)$. Then $\theta_{q y_{n}}(1 / n)>1-(1 / n)$ and $\Psi_{q y_{n}}(1 / n)>1-(1 / n)$ from which $(\theta \vee \Psi)_{q y_{n}} \rightarrow H$. Since $(\theta \vee \Psi)_{p q}$ is left-continuous and $(\theta \vee \Psi)_{p q}(\varepsilon)>$ $1-\lambda$, there exists a $\delta>0$ for which $(\theta \vee \Psi)_{p q}(\varepsilon-\delta)>1-\lambda$. Now $(\theta \vee \Psi)_{p y_{n}}(\varepsilon) \geqq \Delta\left((\theta \vee \Psi)_{p q}(\varepsilon-\delta),(\theta \vee \Psi)_{q y_{n}}(\delta)\right) \rightarrow(\theta \vee \Psi)_{p q}(\varepsilon-\delta)>1-\lambda$ from which one draws the contradiction that $y_{n} \in N(p, \varepsilon, \lambda, \theta \vee \Psi)$ for large $n$.

Proposition 2. Let $\theta, \Psi \in \mathscr{M}(X)$ and suppose $\tau(\theta)$ and $\tau(\Psi)$ are each compact. Then $\tau(\theta \vee \Psi)$ is totally bounded. 
Proof. Let $d_{1}, d_{2}$ be pseudo-metrics on $X$ which determine $\tau(\theta)$ and $\tau(\Psi)$ respectively. Then $D=d_{1}+d_{2}$ is a pseudo-metric for $\tau(\theta \vee \Psi)$. Let $\varepsilon>0$ be given. Let $p_{i}, q_{j} \in X, 1 \leqq i \leqq N, 1 \leqq j \leqq M$, be chosen such that $\bigcup_{i=1}^{N} B\left(X, p_{i}, d_{1}, \varepsilon / 2\right)=\bigcup_{j=1}^{M} B\left(X, q_{j}, d_{2}, \varepsilon / 2\right)=X$. Then it is easy to verify that, for each $i$ and $j, B\left(X, p_{i}, d_{1}, \varepsilon / 2\right) \cap$ $B\left(X, q_{j}, d_{2}, \varepsilon / 2\right) \subset B\left(X, z_{i j}, D, \varepsilon\right) \quad$ for any $z_{i j} \in B\left(X, p_{i}, d_{1}, \varepsilon / 2\right) \cap$ $B\left(X, q_{j}, d_{2}, \varepsilon / 2\right)$ provided this intersection is nonempty.

Theorem 1. Let $\theta, \Psi \in \mathscr{L}(X, T)$ and let $\left(X^{*}, \tau^{*}\right)$ denote the pseudo-metric space completion of $(X, \tau(\theta \vee \Psi))$. Then:

1. $T$ admits a unique extension to a self-homeomorphism $T^{*}$ of $\left(X^{*}, \tau^{*}\right)$.

2. $\theta \vee \Psi$ admits a unique extension to a map $(\theta \vee \Psi)^{*}: X^{*} \times$ $X^{*} \rightarrow \mathscr{D}$

$$
\begin{aligned}
& \text { 3. }(\theta \vee \Psi)^{*} \in \mathscr{C l}\left(X^{*}, T^{*}\right) \\
& \text { 4. } \tau^{*}=\tau\left((\theta \vee \Psi)^{*}\right)
\end{aligned}
$$

Proof. Let $D^{*}$ denote the pseudo-metric on $\left(X^{*}, \tau^{*}\right)$ which extends the pseudo-metric $D$ on $(X, \tau(\theta \vee \Psi))$. Since $\theta \vee \Psi: X \times X \rightarrow \mathscr{D}$ is a uniformly continuous map [8] it can be extended (Cor. 6.2, Ch. 14 of [3]) to a continuous map $(\theta \vee \Psi)^{*}: X^{*} \times X^{*} \rightarrow \mathscr{D}$. The work of Sherwood [11, 12] implies that $(\theta \vee \Psi)^{*} \in \mathscr{C}(X)$ and $\tau\left((\theta \vee \Psi)^{*}\right)=\tau^{*}$. Since $T$ is uniformly continuous on $(X, D)$ there exists an extension $T^{*}$ which is a self-homeomorphism of $\left(X^{*}, \tau^{*}\right)$. Now since $\tau^{*}$ is compact, $(\theta \vee \Psi)^{*} \in \mathscr{C}\left(X^{*}, T^{*}\right)$.

4. Entropy. We begin by investigating the relation among $h(T, \theta \vee \Psi), h(T, \theta)$ and $h(T, \Psi)$. Several lemmas are required.

LEMMA 2. Let $(X, \rho)$ be a compact pseudo-metric space and $T: X \rightarrow X$ be a homeomorphism. Let $\left\{\mathscr{U}_{n}: n \in \boldsymbol{Z}^{+}\right\}$be a sequence of open covers of $X$ satisfying $\rho$-diam $\mathscr{U}_{n} \rightarrow 0$ as $n \rightarrow \infty$. Then $h\left(T, \mathscr{U}_{n}\right) \rightarrow h(T)$.

Proof. Let $\left\{\mathscr{C}_{n_{j}}: j \in Z^{+}\right\}$be a subsequence of the $\left\{\mathscr{U}_{n}\right\}$. Using the Lebesgue covering lemma one can select a subsequence $\left\{m_{j}\right\}$ of the $\left\{n_{j}\right\}$ such that $\mathscr{U}_{m_{j}} \prec \mathscr{U}_{m_{j+1}}$ for $j \geqq 1$. Now applying the Corollary on page 314 of [1] the desired result is obtained.

Lemma 3. Suppose $D$ and $d$ are pseudo-metrics on $X$ satisfying $D \geqq d, \tau(d)$ is compact and $\tau(D)$ is totally bounded. Assume $T$ is a self-homeomorphism of $(X, d)$ and of $(X, D)$. Let $\left\{\mathscr{V}_{n}: n \in Z^{+}\right\}$be a sequence of $\tau(D)$-open covers of $X$ such that $D$-diam $\mathscr{V}_{n} \rightarrow 0$ as $n \rightarrow \infty$ and each $\mathscr{V}_{n}$ possesses a finite subcover of $X$. Then sup $\{h(T, \mathscr{U}): \mathscr{U} \subset \tau(d)\} \leqq \overline{\lim }_{n \rightarrow \infty} h\left(T, \mathscr{\mathscr { N }}_{n}\right)$. 
Proof. Let $\mathscr{W}_{n}$ be a sequence of $\tau(d)$-open covers of $X$ such that $d$-diam $\mathscr{W}_{n} \rightarrow 0$. Then for each $n>0$ there exists an $m \geqq n$ such that $\mathscr{W}_{n} \prec \mathscr{V}_{m}$. Thus $\lim _{n} h\left(T, \mathscr{W}_{n}\right) \leqq \overline{\lim }_{n} h\left(T, \mathscr{V}_{n}\right)$. Now $\sup \{h(T, \mathscr{U}): \mathscr{C} \subset \tau(d)\}=\lim _{n} h\left(T, \mathscr{W}_{n}\right)$.

Lemma 4. Let $\theta, \Psi \in \mathscr{M}(X, T)$ and let $D$ be a pseudo-metric on $X$ for which $\tau(D)=\tau(\theta \vee \Psi)$. For each $\varepsilon>0$ let $\mathscr{U}_{\varepsilon}=\{B(X, p, D, \varepsilon): p \in X\}$. Then $h\left(T^{*},(\theta \vee \Psi)^{*}\right)=\sup _{\varepsilon} h\left(T, \mathscr{U}_{\varepsilon}\right)$.

Proof. Let $\mathscr{U}_{\varepsilon}^{*}=\left\{B\left(X^{*}, p, D^{*}, \varepsilon\right): p \in X\right\}$. Then $\mathscr{U}_{\varepsilon}=\{A \cap X$ : $\left.A \in \mathscr{U}_{\varepsilon}^{*}\right\}$ and $h\left(T^{*},(\theta \vee \Psi)^{*}\right)=\sup _{\varepsilon} h\left(T^{*}, \mathscr{U}_{\varepsilon}^{*}\right)$. It is easy to verify that $N\left(\bigvee_{0}^{K} T^{* i} \mathscr{U}_{\varepsilon}^{*}\right)=N\left(\bigvee_{0}^{K} T^{i} \mathscr{U}_{\varepsilon}\right)$ for all $K \geqq 0$. Consequently $h\left(T^{*}, \mathscr{U}_{\varepsilon}^{*}\right)=h\left(T, \mathscr{U}_{\varepsilon}\right)$ and the lemma is proven.

Theorem 2. Let $\theta, \Psi \in \mathscr{C}(X, T)$. Then:

$\operatorname{Max}(h(T, \theta), h(T, \Psi)) \leqq h\left(T^{*},(\theta \vee \Psi)^{*}\right) \leqq h(T, \theta)+h(T, \Psi)$

Proof. Let $d_{1}$ and $d_{2}$ be pseudo-metrics on $X$ which generate $\tau(\theta)$ and $\tau(\Psi)$ respectively. Then $D=d_{1}+d_{2}$ is a pseudo-metric for $\tau(\theta \vee \Psi)$. Let $\varepsilon_{n}$ be a sequence of positive numbers such that $\varepsilon_{n} \rightarrow 0$. Let $\mathscr{\mathscr { V }}_{n}=\left\{B\left(X, p, D, \varepsilon_{n}\right): p \in X\right\}$ and $\mathscr{\mathscr { N }}_{n}^{*}=\left\{B\left(X^{*}, p, D^{*}, \varepsilon_{n}\right): p \in X\right\}$. Applying lemma 3 , we have $h\left(T^{*},(\theta \vee \Psi)^{*}\right)=\lim _{n \rightarrow \infty} h\left(T^{*}, \mathscr{Y}_{n}^{*}\right) \geqq$ $\varlimsup_{n \rightarrow \infty} h\left(T, \mathscr{V}_{n}\right) \geqq \sup \left\{h(T, \mathscr{C}): \mathscr{U} \subset \tau\left(d_{1}\right)\right\}=h(T, \theta)$.

Let $\mathscr{P}_{n}=\left\{B\left(X, p, d_{1}, 1 / n\right): p \in X\right\}, \quad \mathbb{Q}_{n}=\left\{B\left(X, p, d_{2}, 1 / n\right): p \in X\right\}$ and $\mathscr{R}_{n}=\{B(X, p, D, 1 / n): p \in X\}$. Since $\mathscr{R}_{n} \prec \mathscr{P}_{4 n} \vee \mathscr{Q}_{4 n}$ we have $h\left(T, \mathscr{R}_{n}\right) \leqq h\left(T, \mathscr{P}_{4 n} \vee \mathscr{Q}_{4 n}\right) \leqq h\left(T, \mathscr{P}_{4 n}\right)+h\left(T, \mathscr{Q}_{4 n}\right)$. Lemma 4 yields $h\left(T^{*},(\theta \vee \Psi)^{*}\right)=\lim _{n \rightarrow \infty} h\left(T, \mathscr{R}_{n}\right) \leqq \lim _{n \rightarrow \infty} h\left(T, \mathscr{P}_{4 n}\right)+\lim _{n \rightarrow \infty} h\left(T, \mathscr{Q}_{4 n}\right)=$ $h(T, \theta)+h(T, \Psi)$.

Example 1. Let $Y=\{0,1,2\}$ and $X=Y^{z}$. Define the shift $T: X \rightarrow X$ by $T\left(\left\{y_{i}\right\}\right)=\left\{y_{i+1}\right\}$. Let $d_{1}$ and $d_{2}$ be pseudo-metrics on $X$ given by:

$$
d_{1}\left(\left\{u_{i}\right\},\left\{z_{i}\right\}\right)=\sum_{i=-\infty}^{\infty} \frac{\left|\omega\left(u_{i}\right)-\omega\left(z_{i}\right)\right|}{2^{|i|}}
$$

where

$$
\omega(a)=\left\{\begin{array}{lll}
0 & \text { if } & a=2 \\
1 & \text { if } & a=0,1
\end{array}\right.
$$

and

$$
d_{2}\left(\left\{u_{i}\right\},\left\{z_{i}\right\}\right)=\sum_{i=-\infty}^{\infty} \frac{\left|\alpha\left(u_{i}\right)-\alpha\left(z_{i}\right)\right|}{2^{|i|}}
$$


where

$$
\alpha(\alpha)=\left\{\begin{array}{lll}
0 & \text { if } & a=0 \\
1 & \text { if } & a=1,2
\end{array}\right.
$$

for all $\left\{u_{i}\right\}$ and $\left\{z_{i}\right\} \in X$.

Define $\theta, \Psi \in \mathscr{C}(X, T)$ by:

$$
\theta_{u z}(\varepsilon)=H\left(\varepsilon-d_{1}(u, z)\right)
$$

and

$$
\Psi_{u z}(\varepsilon)=H\left(\varepsilon-d_{2}(u, z)\right) \quad \text { for all } \varepsilon>0 \text { and all } u, z \in X \text {. }
$$

Then it follows that $h\left(T^{*},(\theta \vee \Psi)^{*}\right)=\ln 3, \operatorname{Max}(h(T, \theta), h(T, \Psi))=\ln$ 2 , and $h(T, \theta)+h(T, \Psi)=\ln 4$.

Definition. If $\theta, \Psi \in \mathscr{M}_{F}(X, T)$ let $h_{T}(\theta \mid \Psi)=h\left(T^{*},(\theta \vee \Psi)^{*}\right)-$ $h(T, \Psi)$

Proposition 3. Assume $\theta, \Psi, \Gamma \in \mathscr{M}_{F}(X, T)$. Then:

(a) $0 \leqq h_{T}(\theta \mid \Psi) \leqq h(T, \theta)$

(b) $h_{T}(\theta \mid \theta)=0$

( c ) $h_{T}(\theta \vee \Psi \mid \Gamma)=h_{T}(\theta \mid \Psi \vee \Gamma)+h_{T}(\Psi \mid \Gamma)$ provided $\theta \vee \Psi$, $\Psi \vee \Gamma \in \mathscr{M}_{F}(X, T)$

(d) $h_{T}(\theta \mid \Xi)=h(T, \theta)$

Proof. Statement $(a)$ is a corollary of Theorem 2. Statements (b), (c) and (d) follow quickly from the definitions.

Proposition 4. Let $\theta, \Gamma \in \mathscr{M}_{F}(X, T)$. Suppose $\theta \vee \Gamma \in \mathscr{C}_{F}(X, T)$ and that $(X, \Gamma)$ is a Menger space. Then $h_{T}(\theta \mid \Gamma)=0$.

Proof. This follows from the fact that any two compact metrizable topologies on $X$, each of which renders $T$ a homeomorphism, yield the same topological entropy for $T$.

Proposition 5. Let $\theta, \Psi \in \mathscr{M}_{F}(X, T)$. Assume that $\theta \vee \Psi \in$ $\mathscr{C}_{F}(X, T)$ and that $\theta_{p q}=H$ if and only if $\Psi_{p q}=H$. Then $h_{T}(\theta \mid \Psi)=0$.

Proof. For $x, y \in X$, define $x \sim y$ if and only if $\theta_{x y}=H$. This equivalence relation on $X$ induces a self-homeomorphism $\widetilde{T}$ of $X / \sim$. It is easy to verify that $h(T)=h(\widetilde{T})$. One can then apply Proposition 4.

Proposition 6. Let $\theta, \Psi, \Gamma \in \mathscr{M}_{F}(X, T)$ and suppose $\Psi \leqq \Gamma$. Then $h_{T}(\Psi \mid \theta) \geqq h_{T}(\Gamma \mid \theta)$. 
Proof. Lemma 4 of [7] yields $\tau(\Gamma) \subset \tau(\Psi \vee \theta)$. Since $\tau(\theta) \subset$ $\tau(\Psi \vee \theta)$ we have $\tau(\Gamma \vee \theta) \subset \tau(\Psi \vee \theta)$. Let $\left(X_{1}^{*}, \tau_{1}^{*}\right)$ and $\left(X_{2}^{*}, \tau_{2}^{*}\right)$ denote the completions of $(X, \tau(\theta \vee \Gamma))$ and $(X, \tau(\theta \vee \Psi))$ respectively, and let $T_{1}^{*}$ and $T_{2}^{*}$ denote the extensions of $T$ to $X_{1}^{*}$ and $X_{2}^{*}$ respectively. One may assume that $X_{1}^{*} \subset X_{2}^{*}$ and that $T_{2}^{*}$ extends $T_{1}^{*}$. Then the relative topology on $X_{1}^{*}$ induced by $\tau_{2}^{*}$ contains $\tau_{1}^{*}$. Let $D_{1}$ and $D_{2}$ denote pseudo-metrics on $X_{1}^{*}$ which generate $\tau_{1}^{*}$ and $\left.\tau_{2}^{*}\right|_{x_{1}^{*}}$ (the topology induced on $X_{1}^{*}$ by $\tau_{2}^{*}$ ) respectively. By replacing $D_{2}$ with $D_{1}+D_{2}$, if necessary, we may assume that $D_{1} \leqq D_{2}$. Let $D_{2}^{*}$ denote the extension of $D_{2}$ to $\left(X_{2}^{*}, \tau_{2}^{*}\right)$. Let $\mathscr{V}_{n}^{*}=\left\{B\left(X_{2}^{*}, p, D_{2}^{*}, 1 / n\right)\right.$ : $\left.p \in X_{1}^{*}\right\}$ and $\mathscr{V}_{n}=\left\{A \cap X_{1}^{*}: A \in \mathscr{V}_{n}^{*}\right\}$. Applying Lemma 3 together with the fact that $h\left(T_{1}^{*}, \mathscr{V}_{n}\right)=h\left(T_{2}^{*}, \mathscr{V}_{n}^{*}\right)$, we have:

$$
\begin{aligned}
h\left(T_{1}^{*},(\theta \vee \Gamma)^{*}\right) & \leqq \varlimsup_{n \rightarrow \infty} h\left(T_{1}^{*}, \mathscr{V}_{n}\right) \\
& \leqq \sup _{n} h\left(T_{2}^{*}, \mathscr{V}_{n}^{*}\right) \\
& =h\left(T_{2}^{*},(\theta \vee \Psi)^{*}\right) .
\end{aligned}
$$

ExAMPle 2. Two examples are given to show that, in general, if $\theta, \Psi, \Gamma \in \mathscr{C}_{F}(X, T)$ and $\Psi \leqq \Gamma$ then $h_{T}(\theta \mid \Psi)$ may be less than or greater than $h_{T}(\theta \mid \Gamma)$.

First, note that $\Psi \leqq \Xi$ and $h_{T}(\theta \mid \Xi)=h(T, \theta)$. Thus, if $h(\mathrm{~T}, \theta)>0$, then $0=h_{T}(\theta \mid \theta)<h_{T}(\theta \mid \Xi)$.

We now provide an example in which $\Psi \leqq \Gamma$ and $h_{T}(\theta \mid \Psi)>$ $h_{T}(\theta \mid \Gamma)$. Let $Y=\{1,2,3,4,5\}$ and $X=Y^{z}$. Define three pseudometrics on $X$ as follows:

$$
D\left(\left\{y_{i}\right\},\left\{z_{i}\right\}\right)=\sum_{-\infty}^{\infty} \frac{\left|c\left(y_{i}\right)-c\left(z_{i}\right)\right|}{2^{|i|}}
$$

where

$$
\begin{gathered}
c(a)= \begin{cases}1 & \text { if } a=5 \\
0 & \text { otherwise }\end{cases} \\
d\left(\left\{y_{i}\right\},\left\{z_{i}\right\}\right)=\sum_{-\infty}^{\infty} \frac{\left|s\left(y_{i}\right)-s\left(z_{i}\right)\right|}{2^{|i|}}
\end{gathered}
$$

where

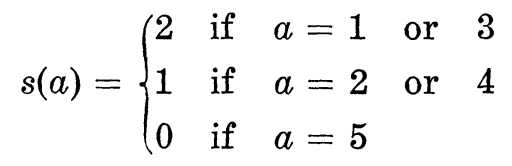

$$
\begin{aligned}
& \rho\left(\left\{y_{i}\right\},\left\{z_{i}\right\}\right)=\sum_{-\infty}^{\infty} \frac{\left|t\left(y_{i}\right)-t\left(z_{i}\right)\right|}{2^{|i|}}
\end{aligned}
$$


where

$$
t(a)= \begin{cases}1 & \text { if } a=1 \text { or } 2 \\ 0 & \text { otherwise }\end{cases}
$$

for all $\left\{y_{i}\right\},\left\{z_{i}\right\} \in X$.

Define $\theta, \Psi, \Gamma \in \mathscr{M}(X)$ as follows:

$$
\begin{aligned}
\theta_{p q}(\varepsilon) & =H(\varepsilon-\rho(p, q)) \\
\Gamma_{p q}(\varepsilon) & =H(\varepsilon-D(p, q)) \\
\Psi_{p q}(\varepsilon) & =H(\varepsilon-d(p, q))
\end{aligned}
$$

for all $p, q \in X$.

Let $T: X \rightarrow X$ be the shift given by $T\left(\left\{y_{i}\right\}\right)=\left\{y_{i+1}\right\}$. Then $\theta, \Gamma, \Psi \in \mathscr{M}(X, T), \Psi \leqq \Gamma, h_{r}(\theta \mid \Psi)=h(T, \theta \vee \Psi)-h(T, \Psi)=\ln 5-$ $\ln 3=\ln 5 / 3$, and $h_{T}(\theta \mid \Gamma)=h(T, \theta \vee \Gamma)-h(T, \Gamma)=\ln 3-\ln 2=$ $\ln 3 / 2$.

TheOREM 3. Let $\theta_{n}, \Psi_{n}, \theta, \Psi \in \mathscr{A}_{F}(X, T)$. Suppose $\theta_{n} \geqq \theta, \Psi_{n} \geqq \Psi$, $\theta_{n} \stackrel{\mathscr{B}}{\rightarrow} \theta, \Psi_{n} \stackrel{\mathscr{G}}{\rightarrow} \Psi$ and $\theta \vee \Psi \in \mathscr{M}(X, T)$. Then $h_{T}\left(\theta_{n} \mid \Psi_{n}\right) \rightarrow h_{T}(\theta \mid \Psi)$ as $n \rightarrow \infty$.

The proof of this theorem is based upon the following lemma.

Lemma 5. Assume $\Sigma \in \mathscr{C}(X, T), \Omega \in \mathscr{C}(X), \tau(\Omega)$ is totally bounded, $\Omega \geqq \Sigma$ and $T$ is a self-homeomorphism of $(X, \tau(\Omega))$. Then $\Omega \in \mathscr{M}(X, T)$.

Proof. We must show $\tau(\Omega)$ is compact. Let $X^{*}$ be the completion of $(X, \tau(\Omega))$ and $T^{*}$ be the extension of $T$ to $X^{*}$. So $\Omega^{*} \epsilon$ $\mathscr{M}\left(X^{*}, T^{*}\right)$. Since $X^{*}$ is compact, Lemma 3 of [7] is applicable. Thus, given $q \in X^{*},\left\{N\left(q, \varepsilon, \lambda, \Omega^{*}\right): \varepsilon, \lambda>0\right\}$ is a local basis for $\tau\left(\Omega^{*}\right)$ at $q$. If $p \in X$ observe that $N\left(p, \varepsilon, \lambda, \Omega^{*}\right) \cap X=N(p, \varepsilon, \lambda, \Omega)$. Hence, for $p \in X,\{N(p, \varepsilon, \lambda, \Omega): \varepsilon, \lambda>0\}$ is a local basis for $\tau(\Omega)$ at $p$. Now an argument analogous to that used in lemma 4 of [7] yields $\tau(\Omega) \subset$ $\tau(\Sigma)$.

Proof of Theorem 3. Since $\theta_{n} \vee \Psi_{n} \geqq \theta \vee \Psi \in \mathscr{M}_{F}(X, T)$ and $\theta_{n} \vee \Psi_{n} \in \mathscr{M}(X)$ the preceding lemma yields $\theta_{n} \vee \Psi_{n} \in \mathscr{C}(X, T)$. Now, applying the main theorem of [7], $h\left(T, \theta_{n} \vee \Psi_{n}\right) \rightarrow h(T, \theta \vee \Psi)$ and $h\left(T, \Psi_{n}\right) \rightarrow h(T, \Psi)$. The desired result now follows.

We conclude with a brief consideration of a special class of pseudo-Menger maps. 
Definition. The map $\theta \in \mathscr{M}(X, T)$ is $(X, T)$-deterministic if $h(T, \theta)=0$.

Proposition 7. Let $\theta, \Gamma \in \mathscr{M}(X, T)$ and suppose that $\theta \geqq \Gamma$. If $\Gamma$ is $(X, T)$-deterministic then so is $\theta$.

Proof. This follows at once from Lemma 4 of [7] where it is shown that $\tau(\theta) \subset \tau(\Gamma)$.

The following two propositions are consequences of Theorem 2.

Proposition 8. Let $\theta, \Gamma \in \mathscr{C l}(X, T)$. Then $\theta$ and $\Gamma$ are $(X, T)$ deterministic if and only if $\theta \vee \Gamma$ is $\left(X^{*}, T^{*}\right)$-deterministic.

Proposition 9. If $\Gamma \in \mathscr{C}(X, T)$ is $(X, T)$-deterministic and $\theta \in \mathscr{M}_{F}(X, T)$ then $h_{T}(\theta \mid \Gamma)=h(T, \theta)$.

ACKNOWLEDGMENT. The author is grateful to the referee for uncovering several errors in the original version of this paper.

\section{REFERENCES}

1. R. L. Adler, A. G. Konheim and M. H. McAndrew, Topological entropy, Trans. Amer. Math. Soc., 114 (1965), 309-319.

2. P. Billingsley, Ergodic Theory and Information, Wiley and Sons, New York, 1965.

3. J. Dugundji, Topology, Allyn and Bacon, Boston, 1967.

4. M. Loève, Probability Theory, 3rd Ed., Van Nostrand Co., Princeton, N. J., 1963.

5. K. Menger, Statistical metrics, Proc. Nat. Acad. Sci., 28 (1942), 535-537.

6. W. Parry, Entropy and Generators in Ergodic Theory, Benjamin, New York, 1969.

7. A. Saleski, Entropy of self-homeomorphisms of statistical pseudo-metric spaces, Pacific J. Math., 51 (1974), 537-542.

8. B. Schweizer, On the uniform continuity of the probabilistic distance, Z. Wahr., 5 (1966), 357-360.

9. B. Schweizer and A. Sklar, Statistical metric spaces, Pacific J. Math., 10 (1960), 313-334.

10. B. Schweizer, A. Sklar and E. Thorp, The metrization of statistical metric spaces, Pacific J. Math., 10 (1960), 673-675.

11. H. Sherwood, On the completion of probabilistic metric spaces, Z. Wahr., 6 (1966), $62-64$.

12. - Complete probabilistic metric spaces, Z. Wahr., 20 (1971), 117-128.

Received October 4, 1974 and in revised form April 21, 1975.

UNIVERSITY OF VIRGINIA 



\title{
PACIFIC JOURNAL OF MATHEMATICS
}

\section{EDITORS}

RichaRd ARENS (Managing Editor)

University of California

Los Angeles, California 90024

\section{R. A. Beaumont}

University of Washington

Seattle, Washington 98105
J. DUGUNDJI

Department of Mathematics University of Southern California Los Angeles, California 90007

D. Gilbarg and J. Milgram

Stanford University

Stanford, California 94305

\section{ASSOCIATE EDITORS}
E. F. BECKENBACH
B. H. NeUmanN
F. WOLF
K. YoshIDA

\section{SUPPORTING INSTITUTIONS}

\author{
UNIVERSITY OF BRITISH COLUMBIA \\ CALIFORNIA INSTITUTE OF TECHNOLOGY \\ UNIVERSITY OF CALIFORNIA \\ MONTANA STATE UNIVERSITY \\ UNIVERSITY OF NEVADA \\ NEW MEXICO STATE UNIVERSITY \\ OREGON STATE UNIVERSITY \\ UNIVERSITY OF OREGON \\ OSAKA UNIVERSITY
}

\author{
UNIVERSITY OF SOUTHERN CALIFORNIA \\ STANFORD UNIVERSITY \\ UNIVERSITY OF TOKYO \\ UNIVERSITY OF UTAH \\ WASHINGTON STATE UNIVERSITY \\ UNIVERSITY OF WASHINGTON \\ AMERICAN MATHEMATICAL SOCIETY
}

The Supporting Institutions listed above contribute to the cost of publication of this Journal, but they are not owners or publishers and have no responsibility for its content or policies.

Mathematical papers intended for publication in the Pacific Journal of Mathematics should be in typed form or offset-reproduced, (not dittoed), double spaced with large margins. Underline Greek letters in red, German in green, and script in blue. The first paragraph or two must be capable of being used separately as a synopsis of the entire paper. Items of the bibliography should not be cited there unless absolutely necessary, in which case they must be identified by author and Journal, rather than by item number. Manuscripts, in triplicate, may be sent to any one of the editors. Please classify according to the scheme of Math. Reviews, Index to Vol. 39. All other communications should be addressed to the managing editor, or Elaine Barth, University of California, Los Angeles, California, 90024.

The Pacific Journal of Mathematics expects the author's institution to pay page charges, and reserves the right to delay publication for nonpayment of charges in case of financial emergency.

100 reprints are provided free for each article, only if page charges have been substantially paid. Additional copies may be obtained at cost in multiples of 50 .

The Pacific Journal of Mathematics is issued monthly as of January 1966. Regular subscription rate: $\$ 72.00$ a year (6 Vols., 12 issues). Special rate: $\$ 36.00$ a year to individual members of supporting institutions.

Subscriptions, orders for back numbers, and changes of address should be sent to Pacific Journal of Mathematics, 103 Highland Boulevard, Berkeley, California, 94708.

PUBLISHED BY PACIFIC JOURNAL OF MATHEMATICS, A NON-PROFIT CORPORATION

Printed at Kokusai Bunken Insatsusha (International Academic Printing Co., Ltd.), 8-8, 3-chome, Takadanobaba, Shinjuku-ku, Tokyo 160, Japan.

Copyright (C) 1975 by Pacific Journal of Mathematics Manufactured and first issued in Japan 


\section{Pacific Journal of Mathematics}

\section{Vol. 59, No. $2 \quad$ June, 1975}

Aharon Atzmon, A moment problem for positive measures on the unit disc ........

Peter W. Bates and Grant Bernard Gustafson, Green's function inequalities for

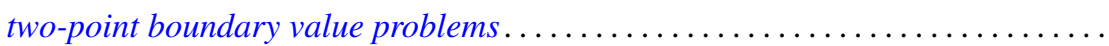

Howard Edwin Bell, Infinite subrings of infinite rings and near-rings ...........

Grahame Bennett, Victor Wayne Goodman and Charles Michael Newman, Norms of

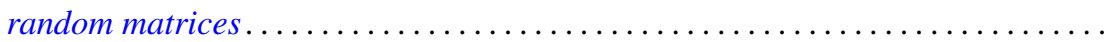

Beverly L. Brechner, Almost periodic homeomorphisms of $E^{2}$ are periodic.........

Beverly L. Brechner and R. Daniel Mauldin, Homeomorphisms of the plane ........

Jia-Arng Chao, Lusin area functions on local fields ......................

Frank Rimi DeMeyer, The Brauer group of polynomial rings ...............

M. V. Deshpande, Collectively compact sets and the ergodic theory of

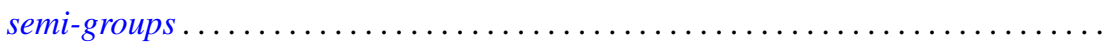

Raymond Frank Dickman and Jack Ray Porter, $\theta$-closed subsets of Hausdorff

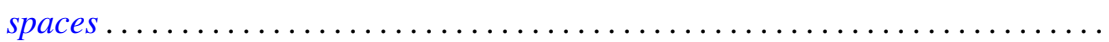

Charles P. Downey, Classification of singular integrals over a local field .......... 407

Daniel Reuven Farkas, Miscellany on Bieberbach group algebras . . . . . . . . . . . .

Peter A. Fowler, Infimum and domination principles in vector lattices . . . . . . . . . .

Barry J. Gardner, Some aspects of T-nilpotence. II: Lifting properties over

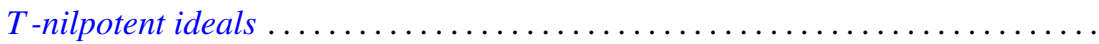

Gary Fred Gruenhage and Phillip Lee Zenor, Metrization of spaces with countable

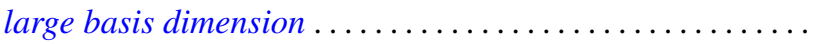

J. L. Hickman, Reducing series of ordinals...

Hugh M. Hilden, Generators for two groups related to the braid group ...

Tom (Roy Thomas Jr.) Jacob, Some matrix transformations on analytic sequence

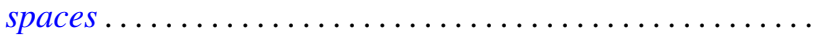

Elyahu Katz, Free products in the category of $k_{w}$-groups . .....

Tsang Hai Kuo, On conjugate Banach spaces with the Radon-Nikodým property...

Norman Eugene Liden, $K$-spaces, their antispaces and related mappings ...

Clinton M. Petty, Radon partitions in real linear spaces ........

Alan Saleski, A conditional entropy for the space of pseudo-Menger maps ....

Michael Singer, Elementary solutions of differential equations .

Eugene Spiegel and Allan Trojan, On semi-simple group algebras. I. . .

Charles Madison Stanton, Bounded analytic functions on a class of open Riemann

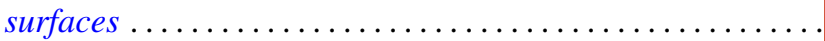

Sherman K. Stein, Transversals of Latin squares and their generalizations ....

Ivan Ernest Stux, Distribution of squarefree integers in non-linear sequences . . .

Lowell G. Sweet, On homogeneous algebras ................

Lowell G. Sweet, On doubly homogeneous algebras .......... .

Florian Vasilescu, The closed range modulus of operators ......

Arthur Anthony Yanushka, A characterization of the symplectic groups $\operatorname{PSp}(2 m, q)$

as rank 3 permutation groups... 